\title{
Performance Improvement for OFDM-RoF Transported 60 GHz System using Spatial Diversity and Multiplexing
}

\author{
Usman Habib $^{1}$, Anthony E Aighobahi ${ }^{1}$, Manish Nair ${ }^{1}$, Huiling Zhu ${ }^{1}$, Terence Quinlan ${ }^{2}$, Stuart D Walker ${ }^{2}$ and Nathan J \\ Gomes $^{1}$ \\ ${ }^{1}$ Communication Research Group, University of Kent, Canterbury, UK \\ ${ }^{2}$ School of Computer Science and Electronic Engineering, University of Essex, UK \\ uh23@kent.ac.uk
}

\begin{abstract}
GHz system architectures with Radio over Fiber (RoF) transport and integrated transmitters/receivers provide a comprehensive solution for future mobile systems. Since $60 \mathrm{GHz}$ communication relies on line-of-sight (LoS) conditions and narrow-beam antennas to compensate the high path-loss, it has limitations in terms of coverage for multiple user locations. In this paper, performance analysis of a $60 \mathrm{GHz}$ integrated transmitter and receiver system supported by RoF transport has been performed experimentally at different user locations for up to 1.5m transmission distance. Extension of experimental results to prove feasibility for longer distances has been shown with a simulation model, whose results at various shorter distances have been benchmarked against the acquired experimental results at different user locations. A modified version of the Saleh Valenzuela channel has been used to model the millimeter wave $(\mathrm{mmW}) \mathrm{LoS}$ indoor experimental environment. Furthermore, as a proof of concept, we present an experimental analysis demonstrating an improvement in performance of the proposed RoF based $60 \mathrm{GHz}$ system using spatial diversity and multiplexing. Channel measurements at different transmitter/receiver locations and their processing have shown that an improvement (decrease from $12.5 \%$ to $10.5 \%$ ) in Error Vector Magnitude (EVM) can be achieved using the Alamouti Space Time Block Coding algorithm. Then it has been shown that a two-fold data rate increase can be obtained by combining data from two transmitter locations using the Zero Forcing algorithm.
\end{abstract}

Keywords-Radio over Fiber, Remote Antenna Unit, Space Time Block Coding, Zero Forcing, Error Vector Magnitude

\section{INTRODUCTION}

The meteoric rise in demand for ever higher data-rates along with lower latencies has created new challenges in existing communication networks [1] which has led to research and development of fifth generation $(5 \mathrm{G})$ cellular networks. For new systems, the severe scarcity of spectrum in the current cellular bands has led to proposals for use of millimeter waves $(\mathrm{mmW})$, where larger bands of frequency are available to offer high data rates, and in particular, the $60 \mathrm{GHz}$ band, which has attracted substantial interest in recent research due to its unlicensed $7 \mathrm{GHz}$ bandwidth [2].

When adopting $60 \mathrm{GHz}$ in indoor environments, the integration of mmW front end wireless links with Radio-overfiber transmission is an attractive approach [3]. Properties of optical fiber communication such as ultra-high capacity and low loss propagation make it easy to realize a robust architecture for data transport between the Central Unit (CU) and the Remote Antenna Unit (RAU). The RoF part serves as a backbone for the whole network and provides centralized control [4]. RAU communication with the mobile user (MU) at $60 \mathrm{GHz}$ can then be achieved using compact integrated transmitter and receiver units. Since $60 \mathrm{GHz}$ wireless links rely on line-of-sight (LoS) conditions with narrow-beam antennas to compensate for the high path-loss [5], the coverage for multiple MU locations is still a challenge for $60 \mathrm{GHz}$ communication systems that needs to be addressed, and has been highlighted in recent research [6]. Theoretical analysis has been presented for the indoor coverage of $60 \mathrm{GHz}$ systems [7] and the use of repeaters has been proposed to address the coverage issue [8]. However experimental implementations for $60 \mathrm{GHz}$ transmission which show coverage improvement are lacking. Spatial diversity and spatial multiplexing techniques have been widely deployed in lower-frequency systems, such as IEEE 802.11n/ac and LTE 4G standards, to improve the reliability and/or increase the data rate of the system [9]. These can provide coverage improvement for a $\mathrm{mmW}$ communication system by making use of the decorrelation of different channel paths from spatially distributed transmitting units. We have previously shown that for a communication system based on photonic generation of a $25 \mathrm{GHz}$ carrier, the use of Alamouti Space Time Block Coding STBC can provide improvement in Error Vector Magnitude (EVM) performance, while higher data rates through multiplexing can be achieved with the use of the Zero Forcing algorithm [10].

In this paper, a proof of concept to improve the performance of $60 \mathrm{GHz}$ transmission to multiple user locations in indoor environments by using spatial diversity and spatial multiplexing is demonstrated by extending this previous work [10]. The experimental setup consists of OFDM-RoF transport and upconversion to $60 \mathrm{GHz}$ using an integrated transmitter unit. Wireless transmission through $0.5 \mathrm{~m}, 1 \mathrm{~m}$ and $1.5 \mathrm{~m}$ channels and performance analysis has been conducted at different user locations. The results have been extended to longer distances through simulations by modelling the experimental $\mathrm{mmW}$ environment. To perform theoretical analysis for $\mathrm{mmW}$ transmission systems, channel modeling at $60 \mathrm{GHz}$ has always been a challenge due to high path-loss and larger bandwidth; conventional channel models cannot be applied since they are 


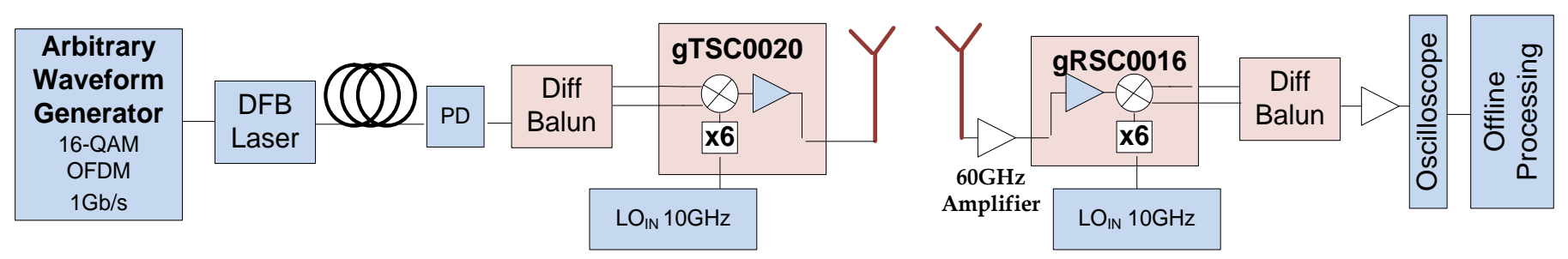

Fig. 1 System model for OFDM-RoF based 60GHz Transmission using Integrated Transmitter/Receiver

based on assumptions of narrow bandwidth. The propagation model presented here uses a modified Saleh Valenzuela (SV) [11] channel which accurately models a wide bandwidth channel and is used to analyze the system performance at longer distances. Furthermore, in order to experimentally analyze the performance of Alamouti STBC and Zero Forcing algorithms, the channel estimation at various transmitter and receiver locations is performed. To accomplish this, OFDM preambles (for channel estimation) and pilot tones (for phase tracking) were used to calculate channel coefficients for two transmitter positions and two receiver positions. From these experiments, it has been shown that performance at different user locations can be improved by reducing the EVM to below the threshold value (i.e. $12.5 \%$ for LTE) through spatial diversity using STBC processing. Moreover, it has also been demonstrated that multiple user data streams transmitted from different transmitter locations can be combined to obtain twice the data rate using spatial multiplexing through the Zero Forcing (ZF) algorithm.

The paper is organized as follows. Section II presents the experimental setup and its performance. The results have been extended for larger coverage areas using a simulation model in Section III. The geometrical arrangement of the experimental setup to achieve spatial diversity and multiplexing is explained in Section IV along with the results. The paper is concluded in Section V.

\section{EXPERIMENTAL SETUP}

Fig. 1 shows the experimental setup in which a Distributed Feedback (DFB) Laser (Emcore 1935F) is modulated by an OFDM signal (at an IF of $1.5 \mathrm{GHz}$ and data rate of $1 \mathrm{~Gb} / \mathrm{s}$ ) generated through a Tektronix 7122C Arbitrary Waveform Generator. The resultant signal is transmitted through a single mode optical fiber to the Central Unit CU where a photodiode (Appointech, 2.5Gbps InGaAs PIN, 0.7A/W) at the other end of the fiber converts the optically modulated signal to an IF signal at the RAU. The system parameters for the experiment can be summarized as in Table. I.

Table I: System Parameters for the experimental setup

\begin{tabular}{|c|c|c|}
\hline Description & Unit & Value \\
\hline Operating Frequency & $\mathrm{GHz}$ & 60 \\
\hline OFDM Signal Bandwidth & $\mathrm{MHz}$ & 305 \\
\hline Modulation & QAM & 16 \\
\hline Tx Sampling Rate & $\mathrm{GSps}$ & 12 \\
\hline Tx-Rx Separation & $\mathrm{m}$ & 1.5 \\
\hline $\mathrm{E}_{\mathrm{b}} / \mathrm{N}_{0}$ Tx SNR & $\mathrm{dB}$ & 30 \\
\hline
\end{tabular}

The IF signal from the photodiode is passed through a differential balun and DC blocker to be upconverted to $61.5 \mathrm{GHz}$ using a gTSC0020 integrated transmitter with an LO of $10 \mathrm{GHz}$. The $61.5 \mathrm{GHz}$ data modulated signal is transmitted over $1.5 \mathrm{~m}$ wireless distance using an $18 \mathrm{dBi}$ gain horn antenna. At the other end, the mmW signal is received by a $16.8 \mathrm{dBi}$ slot antenna [12] and is downconverted to IF using a gRSC0016 integrated receiver using an $\mathrm{LO}$ of $10 \mathrm{GHz}$. The signal is captured using a Tektronix Digital Oscilloscope DPO72304DX for offline processing which includes carrier synchronization and demodulation for EVM analysis. Fig. 2 shows the performance of the experimental RoF transport from the central unit to RAU (without wireless transmission or $60 \mathrm{GHz}$ upconversion) for different input IF power levels which indicates the input power levels for which optimum performance can be achieved through the initial RoF transport of the setup.

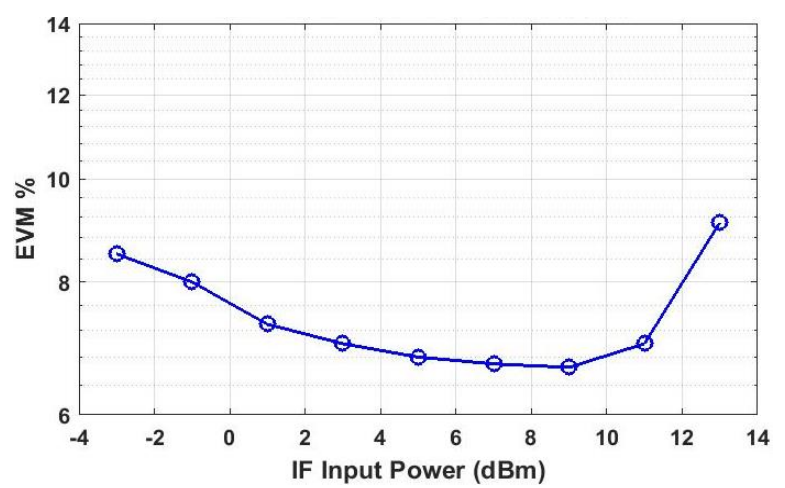

Fig. 2: Performance of RoF transport for different IF input power levels

The performance of the complete system including $60 \mathrm{GHz}$ wireless transmission versus the power of the data signal from the photodiode after RoF transport is shown in Fig. 3. This

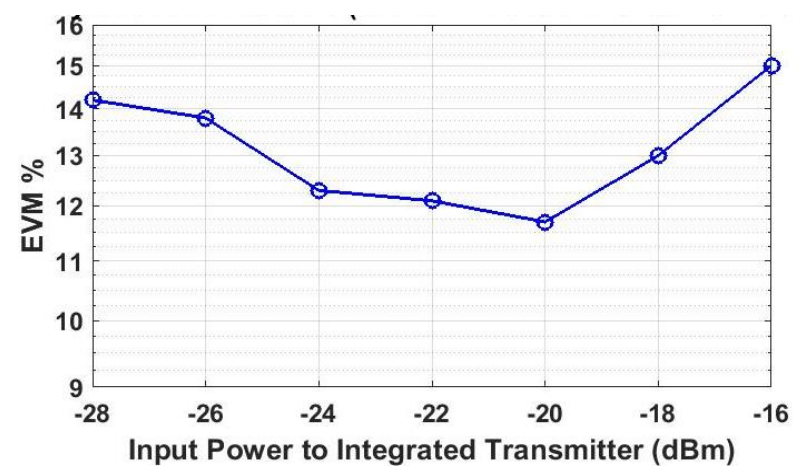

Fig. 3: System Performance of the RoF based $60 \mathrm{GHz}$ Transmission for different IF power levels 
shows that the EVM was found to be less than the $12.5 \%$ limit of LTE (for 16-QAM) for the overall system when the receiver was in LoS and in front of the transmitter as indicated by position $\mathrm{C}$ in geometric arrangement of the experimental setup in Fig. 4. Then, the measurements were performed for five receiver locations (A to $\mathrm{E}$ ) as shown in Fig. 4 which correspond to angular locations of approximately $-15^{\circ},-7.5^{\circ}, 0^{\circ}, 7.5^{\circ}$ and $15^{\circ}$ from geometrical measurements. The transmission distance in the experimental analysis was constrained to $1.5 \mathrm{~m}$ due to lack of availability of $60 \mathrm{GHz}$ band amplifier at the transmitter side.

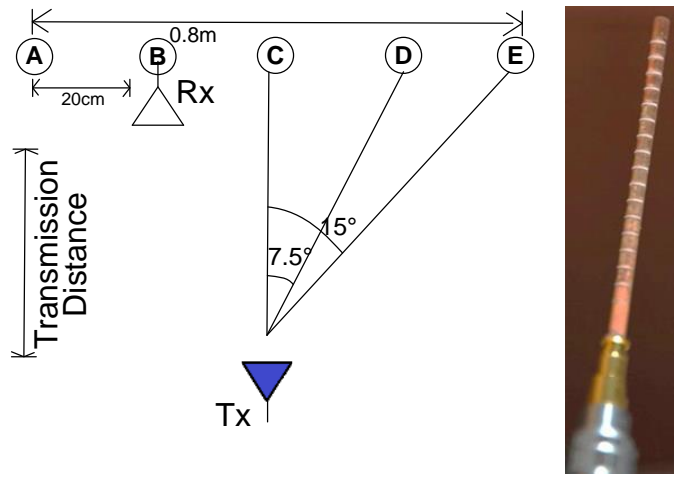

Fig. 4: Geometric orientation of the Experimental Setup and slot antenna array

A simulation model was developed based on the experimental results through the modelling of the $60 \mathrm{GHz}$ indoor experimental environment. The simulation results showed a high degree of agreement with the experimental results for multiple transmission distances of $0.7 \mathrm{~m}, 1 \mathrm{~m}$ and $1.5 \mathrm{~m}$ and for the five receiver locations. Further simulations were performed to show the feasibility of the system for longer distances, which are presented in the next section.

\section{EXTENSION OF EXPERIMENTAL RESULTS USING WIDEBAND MMW CHANNEL MODEL SIMULATIONS}

In order to extend the experimental results of the $60 \mathrm{GHz}$ transmission in the previous section to longer transmission distances, simulation of the indoor experimental environment by applying a LoS extension to the wideband modified Saleh Valenzuela (SV) channel impulse response (CIR) [13] has been considered. The modified SV channel model accurately represents wideband fading in terms of clustered multi-paths which is common in wideband $\mathrm{mmW}$ propagation environments, and hence has been chosen over other channel models, such as Rayleigh or Rician channel which are valid only for narrowband propagation $[11,13]$. Furthermore, other channel models such as WINNER 2, which have been previously used at $\mathrm{mmW}$, are also narrowband and hence cannot be applied to our $305 \mathrm{MHz}$ bandwidth system. Firstly, the coefficients of the wideband modified SV channel, for $v$-th cluster and $u$-th resolvable path of that cluster, are given by:

$$
\alpha_{l}(t)=\sum_{v=0}^{V-1} \sum_{u=0}^{U-1} \sqrt{P_{u v}} \alpha_{u v} \delta\left(t-\tau_{v}-\tau_{u v}\right)
$$

$$
=\sum_{l=0}^{L-1} \sqrt{P_{l}} \alpha_{l} \delta(t-l \tau)
$$

where $\alpha_{1}$ is the receiver CIR of the $l$-th resolvable multi-path signal component from the transmit antenna and $P_{l=u v}$ is the power of the $u$-th resolvable path in the $v$-th cluster. Here $1 \leq l \leq L$, where $\mathrm{L}$ is the total number of resolvable multi-path components, $\mathrm{V}$ denotes the number of clusters, $\mathrm{U}$ the number of resolvable paths in one cluster, and $L=U V$ is the total number of resolvable multi-paths at the receiver [14]. $l$ is related to $u$ and $v$ by $l=v U+u$. In (1) $\alpha_{u v}=\left|\alpha_{u v}\right| \mathrm{e}^{j \theta_{u v}}$ represents the fading of the $u$-th resolvable path in the $v$-th cluster connecting the transmit antenna to the receiver. $\tau_{v}$ is the time-of-arrival (ToA) of the $v$-th cluster and $\tau_{u v}=u \tau$ denotes the ToA of the $u$ th resolvable path in the $v$-th cluster. In our mmW channel, it is assumed that the average power of a multi-path at a given delay is related to the power of the first resolvable multi-path of the first cluster through the following relationship:

$$
P_{u v}^{k}=P_{00}^{k} \exp \left(-\frac{T_{v}}{u}\right) \exp \left(-\frac{T_{u v}}{u}\right)
$$

where $P_{u v, k}=P_{l}=\left|\alpha_{u v}\right|^{2}$ represents the expected power of the $u$-th resolvable path in the $v$-th multipath cluster connecting the user to the transmit antenna. $\Psi$ and $\psi$ are the corresponding power delay constants of the cluster and the resolvable multipath respectively. For the channel model to be generic, we assume that the delay spread, which is $(L-1) \tau$ of the $\mathrm{mmW}$ channel spans $g \geq 1 \quad$ data bits, satisfying $(g-1) N_{\tau} \leq(L-1) \tau \leq g N_{\tau}$, where $N_{\tau}$ is the number of time slots per symbol. Secondly, we assume that the $L$ resolvable multi-path components are randomly distributed and does not change over each symbol. Due to the wide bandwidth at $\mathrm{mmW}$, all the $L$ multi-path components can be resolved at the receiver (Rx) [14]. In the LoS extension for modeling directional antennas, it can be assumed that the first multi-path is the dominant component. A high rate of power decay in (2) will make all the other multi-path coefficients of the wideband SV channel close to zero. The parameters for generating the 3D mmW modified SV channel model at $60 \mathrm{GHz}$ are given in Table II [15].

Table II: 3D mmW Modified SV Channel Parameters [15]

\begin{tabular}{|c|c|c|}
\hline Description & Unit & Value \\
\hline Inter-cluster inter-arrival rate & $1 / \mathrm{ns}$ & 0.21 \\
\hline Inra-cluster inter-arrival rate & $1 / \mathrm{ns}$ & 0.77 \\
\hline Inter-cluster decay factor & $\mathrm{ns}$ & 4.19 \\
\hline Intra-cluster decay factor & $\mathrm{ns}$ & 1.07 \\
\hline Small-scale fading RMS & $\mathrm{dB}$ & 1.26 \\
\hline Inter-cluster Rician K-factor & $\mathrm{dB}$ & -10 \\
\hline Intra-cluster Rician K-factor & $\mathrm{dB}$ & -10 \\
\hline
\end{tabular}

The comparison between experimental results and simulations is presented in Fig. 5 and shows agreement for various transmission distances and receiver locations which 
demonstrates the accuracy of the modelling. Based on this observed agreement between the simulated and measured results, the EVM at various angular locations for longer transmission distances is shown in Fig. 6. The results were obtained by assuming $6 \mathrm{~dB}$ amplification for $4.5 \mathrm{~m}$ and $10 \mathrm{~dB}$ amplification for $10 \mathrm{~m}$ distance (with $2 \mathrm{~dB}$ noise figure for both cases) of the transmit power. The simulation for $4.5 \mathrm{~m}$ distance indicates the practicability of the system in rooms, and at $10 \mathrm{~m}$ for larger transmission distances such as in shopping malls or airports where access points are usually installed on higher structures.

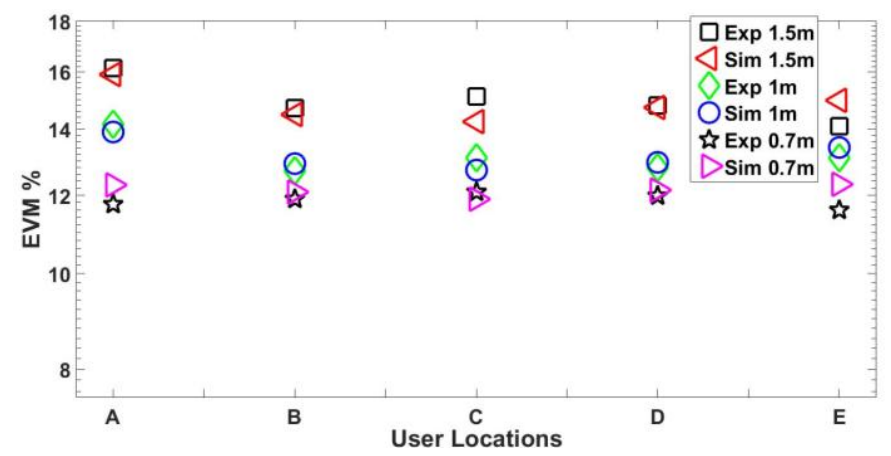

Fig. 5. EVM of simulated and experimental analysis at different angular locations of the user

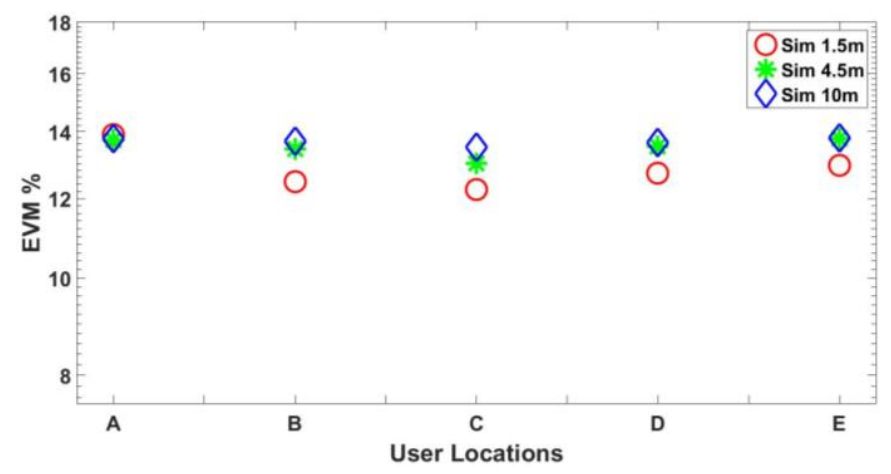

Fig. 6. Simulated EVMs at Different User Locations

From these results, the average spectral efficiency (in $\mathrm{b} / \mathrm{s} / \mathrm{Hz}$ ) at $10 \mathrm{~m} \mathrm{Tx}-\mathrm{Rx}$ separation distance has been calculated and plotted in Fig.7 at five user locations. In order to obtain the average spectral efficiency, the Rx SNR has been first calculated using

$$
S N R_{R x}=\left(E_{b} / N_{0}+\operatorname{SINR}_{i}\right) \cdot C / B \cdot K / P_{l}
$$

where the free space path loss $P_{l}$ at $60 \mathrm{GHz}$, given by

$$
P_{l}=d \cdot f_{c} \cdot 4 \pi / c \sim 66 \mathrm{~dB} \text { at } f_{c}=60 \mathrm{GHz}
$$

In (3), $E_{b} / N_{o}$ is the Tx SNR as mentioned in Table I, $\mathrm{SINR}_{i}$ is the SINR of the $i$-th beam obtained from modelling the system along with the wideband $\mathrm{mmW}$ modified SV channel, $C$ is the Tx sampling rate, $B$ is the channel bandwidth (both are indicated in Table I), $K=4$ is the modulation index for 16QAM. In (4), $d=10 m$ is the Tx-Rx separation, $f c=60 G H z$ is the carrier frequency, and $c$ is the velocity of light. Spectral efficiency is then calculated as:

$$
S E=\log _{2}\left(1+S N R_{R x}\right)
$$

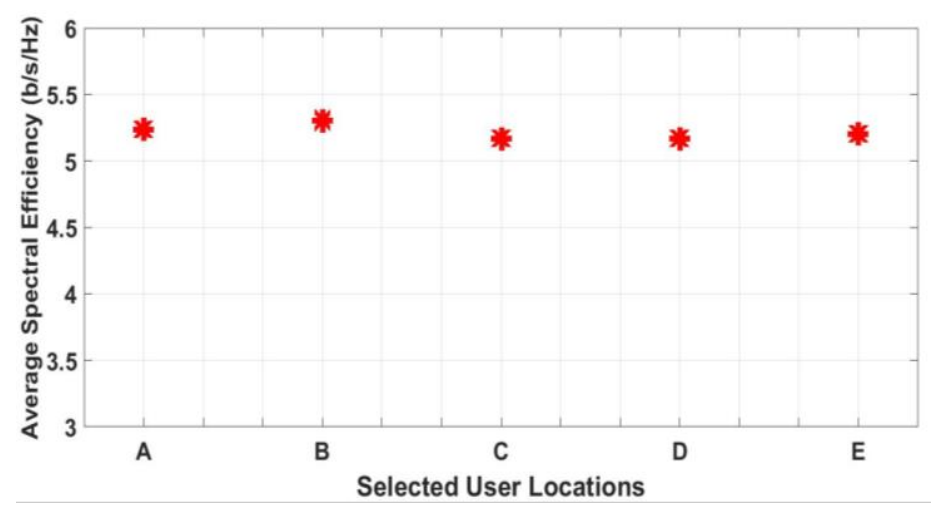

Fig. 7. Average Spectral Efficiency at selected user locations for Tx-Rx separation of $10 \mathrm{~m}$

The average spectral efficiency in Fig. 7 has been obtained over 15 simulation runs to characterize the effect of the randomized Additive White Gaussian Noise (AWGN). It can be observed in Fig. 7 that the average spectral efficiency obtained is just over $5 \mathrm{~b} / \mathrm{s} / \mathrm{Hz}$, when the system parameters in Table I have been applied. The standard deviation was found to be 0.1 for the peak values of the spectral efficiency at these particular user locations.

\section{EXPERIMENTAL ACHIEVEMENT OF SPATIAL DIVERSITY AND SPATIAL MULTIPLEXING}

In this section, we demonstrate performance improvement at the previously discussed user locations and transmission distances through spatial diversity and spatial multiplexing. This can be achieved by measuring the channel transfer function for spatially distributed $N$ transmit and $M$ receive units and combining it with the transmitted symbols using STBC Alamouti or Zero Forcing algorithms. This arrangement can be represented as:

$$
\left[\begin{array}{l}
y_{1} \\
y_{2}
\end{array}\right]=\left[\begin{array}{ll}
h_{11} & h_{12} \\
h_{21} & h_{22}
\end{array}\right]\left[\begin{array}{l}
x_{1} \\
x_{2}
\end{array}\right]+\left[\begin{array}{l}
n_{1} \\
n_{2}
\end{array}\right]
$$

where $y_{1}$ and $y_{2}$ are the received OFDM symbols for $\mathrm{Rx} 1$ and $\mathrm{Rx} 2, x_{1}$ and $x_{2}$ are the transmitted symbols from Tx1 and Tx2, $h_{11}, h_{21}, h_{12}, h_{22}$ are the measured channel transfer functions and $n_{1}, n_{2}$ represent the noise. In the first scenario, in order to achieve spatial diversity using the Alamouti STBC algorithm, the encoded signals are transmitted from two transmit locations over two symbol periods. The first period consists of two symbols $x_{1}$ and $x_{2}$, simultaneously transmitted, while during the second period, these symbols are transmitted again in the form of $-x_{2} *$ from the first antenna and $x_{1} *$ from the second antenna. The Alamouti STBC decoder restores the transmitted symbol periods using (7) and (8):

$$
\begin{gathered}
x_{1}=h_{11} y_{1}+h_{12} y_{2}^{*}+h_{21} y_{1}+h_{22} y_{2}^{*} \\
x_{2}=-h_{11} y_{2}^{*}+h_{12}^{*} y_{1}-h_{22} y_{2}^{*}+h_{21} y_{1}^{*}
\end{gathered}
$$


where $y_{1}$ and $y_{2}$ are the received signals over two symbol periods and $h_{11}, h_{21}, h_{12}, h_{22}$ represent the measured channel co-efficient estimated from the transmitted preamble symbols. In the second case, to achieve multiplexing gain using the zero-forcing algorithm, two different symbols $x_{1}$ and $x_{2}$ are simultaneously transmitted on two different antennas and the symbols are multiplexed using the zero-forcing algorithm. The zero-forcing algorithm uses (9) to restore the separate data where the weight matrix treats all transmitted signals as interference except for the desired signals from the target transmit antennas.

$$
W_{Z F}=\left(H^{H} H\right)^{-1} H^{H}
$$

The complex channel transfer matrix measurements can be obtained by placing transmit antennas at two distributed locations and capturing the data at two receiver location. In order to achieve improvement in performance, the channel transfer function matrix $\left[\begin{array}{ll}h_{11} & h_{12} \\ h_{21} & h_{22}\end{array}\right]$ was experimentally obtained by selecting two positions for the transmit antenna and marking them with a tape according to the layout shown in Fig 8. The coefficient $h_{11}$ was measured by placing the transmit antenna in position Tx1 and receive antenna in position Rx1 while $h_{12}$ was measured by moving the receive antenna to $\mathrm{Rx} 2$ (while keeping the transmit antenna at the same position of Tx1). Subsequently, to measure $h_{21}$, the transmit antenna was moved to Tx2 with receiving antenna at $\mathrm{Rx} 1$ and lastly, $h_{22}$ was measured by moving the received antenna to $\mathrm{Rx} 2$ while keeping the transmit antenna at $\mathrm{T} \times 2$. These measurements were repeated for different user locations (A-E) over a span of $0.8 \mathrm{~m}$ at wireless distance of $1.5 \mathrm{~m}$ (Fig.8). The experiment was conducted for different separation of $20 \mathrm{~cm}, 30 \mathrm{~cm}$ and $40 \mathrm{~cm}$ between $\mathrm{Tx} 1$ and $\mathrm{Tx} 2$ points as shown in Fig. 8.

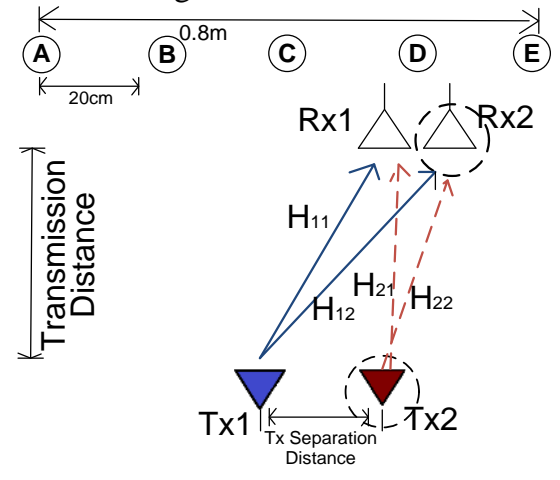

Fig. 8: Experimental Arrangement of Tx and Rx at Different Positions to achieve Spatial Diversity and Multiplexing

The values of $h_{11}, h_{21}, h_{12}, h_{22}$ are used by (7) and (8) in MATLAB/Simulink software for offline processing using the Alamouti STBC algorithm. The EVM obtained at the five user locations (A to E) was improved using the STBC Alamouti signal processing algorithm for the received OFDM symbols $\left(y_{1}\right.$ and $y_{2}$ ) as shown by the results in Fig. 9 where EVM for STBC processed output is less than SISO transmission and is under $12.5 \%$ (16-QAM limit for LTE) for each user location. STBC improves the transmission performance by making use of its orthogonal codes design and decorrelation of the received signals from multiple transmit locations. Thus, the achieved spatial diversity results in enhancement of coverage at multiple user locations.

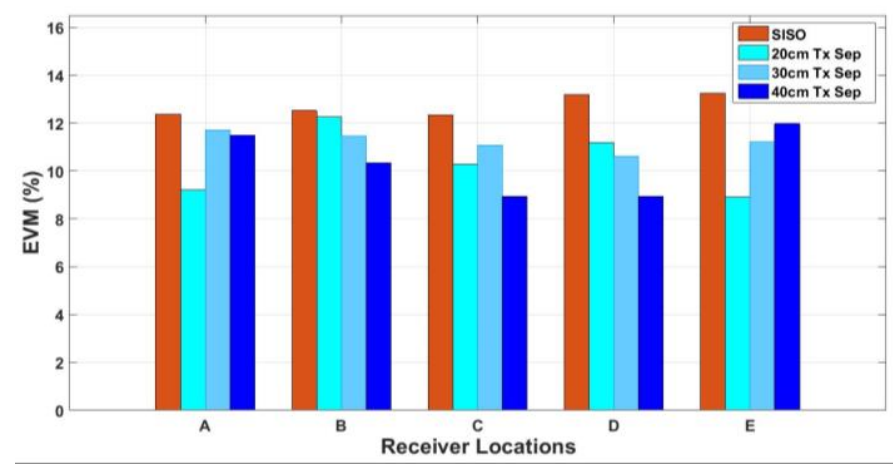

Fig. 9. SISO Demodulation versus STBC Alamouti Processing at Different User Locations

Additionally, the setup was used to realize multiplexing gains by combining the signals from two transmitter locations. One data stream with $0.5 \mathrm{~Gb} / \mathrm{s}$ data rate is transmitted through location Tx1 in the first step and the data is captured at points $\mathrm{Rx} 1$ and $\mathrm{Rx} 2$ by moving the receiving antenna to the respective positions. Then, a second (different) data stream is transmitted through the same transmit antenna from location Tx2 and data was captured again at positions $\mathrm{Rx} 1$ and $\mathrm{Rx} 2$ as given by (9). The captured data is processed using the Zero Forcing algorithm to achieve an aggregate $1 \mathrm{~Gb} / \mathrm{s}$ data rate. Fig. 10 shows that the EVM of multiplexed data with combined data rate is under $12.5 \%$ EVM limit similar to the the single data stream. This verifies the achievement of multiplexing gain with the proposed system at $60 \mathrm{GHz}$ while keeping the spacing between points $\mathrm{Tx} 1$ and $\mathrm{Tx} 2$ as $20 \mathrm{~cm}, 30 \mathrm{~cm}$ and $40 \mathrm{~cm}$.

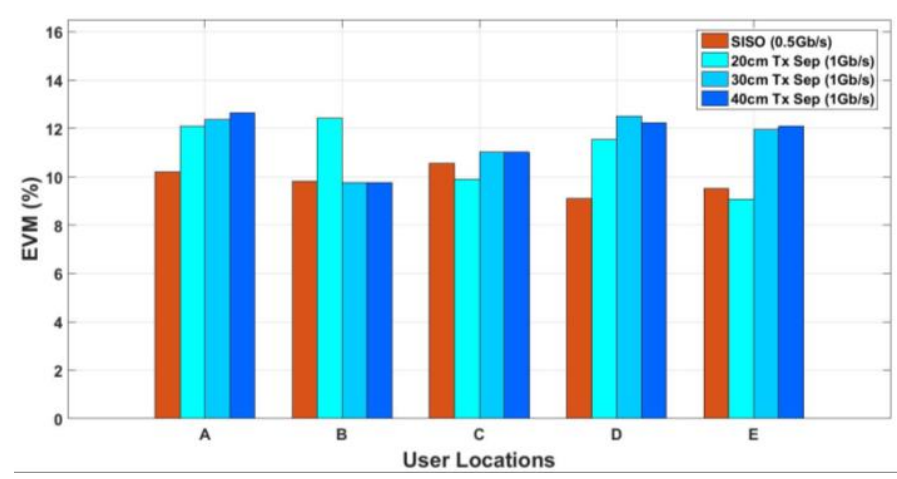

Fig. 10. SISO Demodulation $(0.5 \mathrm{~Gb} / \mathrm{s}$ data rate) versus Zero Forcing Processing (with multiplexed data rate of $1 \mathrm{~Gb} / \mathrm{s}$ )

\section{CONCLUSIONS}

In this paper, the feasibility and analysis of an OFDM-RoF transported $60 \mathrm{GHz}$ system was presented and experimentally demonstrated for up to $1.5 \mathrm{~m}$ transmission distance. A simulation model using a $\mathrm{mmW}$ modified $\mathrm{SV}$ channel was presented, that accurately modelled the $60 \mathrm{GHz}$ experimental EVM results. The feasibility of the experimental setup for longer wireless distances was shown by extension of simulation results. Experimental results using offline processing, in order 
to achieve improvement in performance and data rate, show that the channel estimation and use of the channel transfer function at different transmitter and receiver locations provided spatial diversity and multiplexing gain with Alamouti STBC and ZeroForcing algorithms, respectively.

\section{ACKNOWLEDGMENT}

This work has been supported by the European Union's Horizon 2020 Research and Innovation programme under contract 643297 (RAPID), 644526 (iCIRRUS) and the UK Engineering and Physical Sciences Research Council's NIRVANA project (Reference No. EP/L026031/1).

\section{REFERENCES}

[1] M. Agiwal, A. Roy, N. Saxena, "Next generation 5G wireless networks: a comprehensive survey", in IEEE Communications Survays and Tutorials, Vol. 18 (3), 2016.

[2] S. Jiang, J. Peng, Z. Liu, J. Jiao, S. Jiang, "802.11ad Key Performance Analysis and Its Application in Home Wireless Entertainment", $17^{\text {th }}$ IEEE Conference on Computational Science and Engineering CSE, 2014, pp. 1595-1598.

[3] M. Zhu, L. Zhang, J. Wang, L. Cheng, C. Liu, G. K. Chang, "Radio-overfiber access architecture for integrated broadband wireless services", Journal of Lightwave Technology, 31(23), 2013, pp.3614-3620.

[4] L. Cheng et al. "Coordinated Multipoint Transmissions in MillimeterWave Radio-Over-Fiber Systems." Journal of Lightwave Technology, 34(2), 2016, pp.653-660.

[5] J. Guillory. et al, "Radio over fiber tunnel for $60 \mathrm{GHz}$ wireless home network." In Optical Fiber Communication Conference, p. OWT6. Optical Society of America, 2011.

[6] P. Liu, M. D. Renzo, A. Springer, "Line-of-Sight Spatial Modulation for Indoor mmWave Communication at $60 \mathrm{GHz}$." IEEE Transactions on Wireless Communications 15 (11), 2016, pp.7373-7389.
[7] Y. Li, G. Wang, S. Li, F. Chen, Y. Liu, P. Wang, "Simulation and analysis of $60 \mathrm{GHz}$ millimeter-wave propagation characteristics in indoor complex environment.", IEEE International Conference on. Computational Electromagnetics (ICCEM), 2016, pp.278-280.

[8] J. Shen, D. Zhu, B. Li, P. Liang, "Repeater-enhanced millimeter-wave systems in multi-path environments." IEEE 26th Annual International Symposium on.Personal, Indoor, and Mobile Radio Communications (PIMRC), 2015, pp.769-774.

[9] IEEE 802.11-1999, IEEE Standard for Local and Metropoltian Area Networks - Wireless LAN Medium Access Control (MAC) and Physical Layer (PHY) Specifications, ISO/IEC 8802-11:1999 (E), 1999.

[10] U. Habib. U, A.E.Aighobahi, C.Wang, N.J.Gomes, "Radio over Fiber Transport of mm-Wave $2 \times 2$ MIMO for Spatial Diversity and Multiplexing", International topical meeting on Microwave Photonics (MWP), 2016, Long Beach CA, USA.

[11] O. E. Ayach, S. Rajagopal, S. Abu-Surra, Z. Pi, R. Heath, "Spatially Sparse Precoding in Millimeter Wave MIMO Systems", IEEE Transactions on Wireless Communications, vol. 13 (3) ,2014 , pp.14991513.

[12] T. Quinlan, S. Walker. "A $16.8 \mathrm{dBi}$ quasi-discoidal radiation pattern antenna array for $60 \mathrm{GHz}$ non-line-of-sight applications.", Antennas and Propagation Conference (LAPC), 2014 Loughborough, 2014, pp.210213.

[13] Q. Z. Ahmed, L. L. Yang, "Reduced-rank adaptive multiuser detection in hybrid direct-sequence time-hopping ultrawide bandwidth systems," IEEE Transactions on Wireless Communications, vol. 9 (1), 2010, pp. 156-167.

[14] A. Alkhateeb, R. W. Heath, "Frequency selective hybrid precoding for limited feedback millimeter wave systems", IEEE Transactions on Communications, vol. 64(5), 2016, pp. 1801-1818.

[15] H. Xu, V. Kukshya, T. Rappaport, "Spatial and Temporal Characteristics of $60-\mathrm{GHz}$ Indoor Channels," IEEE Journal on Selected Areas in Communications, vol. 20,(3), 2002, pp. 620-630. 